\title{
Investigate Micropolar Fluid Behavior on MHD Free Convection and Mass Transfer Flow with Constant Heat and Mass Fluxes by Finite Difference Method
}

\author{
Lasker Ershad Ali, Ariful Islam, Nazmul Islam \\ Mathematics Discipline, Khulna University, Khulna, Bangladesh \\ Email address: \\ ershad@math.ku.ac.bd (L. E. Ali), is_arif@yahoo.com (A. Islam), islam_nazmul@rocketmail.com (N. Islam)
}

\section{To cite this article:}

Lasker Ershad Ali, Ariful Islam, Nazmul Islam. Investigate Micropolar Fluid Behavior on MHD Free Convection and Mass Transfer Flow with Constant Heat and Mass Fluxes by Finite Difference Method. American Journal of Applied Mathematics.

Vol. 3, No. 3, 2015, pp. 157-168. doi: 10.11648/j.ajam.20150303.23

\begin{abstract}
Micropolar fluid behavior on MHD free convection and mass transfer with constant heat and mass fluxes is studied numerically. Finite difference technique is used as the main tool for the numerical approach. Micropolar fluid behavior on MHD steady free convection and mass transfer with constant heat and mass fluxes have been considered and its similarities solution have been obtained. Similarity equations of the corresponding momentum, angular momentum, temperature and concentration equations are derived by employing the usual similarity technique. The dimensionless similarity equations for momentum, angular momentum, temperature and concentration equations solved numerically by explicit finite difference technique. With the help of graphs the effects of the various important parameters entering into each of the problems on the velocity, microrotation, temperature and concentration profiles within the boundary layer are separately discussed.
\end{abstract}

Keywords: Micropolar Fluid, Finite Difference Method, Mass Transfer and MHD Free Convection

\section{Introduction}

The concept of micropolar fluid deals with a class of fluids that exhibit microscopic effects arising from the local structure and micro motions of the fluid elements. These fluids contain dilute suspension of rigid macromolecules with individual motions that support stress \& body moments and are influenced by spin inertia. Micropolar fluids are those, which contain micro-constituents that can undergo rotation, the presence of which can affect the hydrodynamics of the flow so that it can be distinctly non-Newtonian. It has many practical applications, for example analyzing the behavior of exotic lubricants, the flow of colloidal suspensions or polymeric fluids, liquid crystals, additive suspensions, human $\&$ animal blood, turbulent shear flow \& so forth. The theory of micropolar fluids was proposed by Eringen in 1966. In this theory, the local effects arising from the microstructure and the intrinsic motion of the fluid elements are taken into account. Physically, the micropolar fluid can consist of a suspension of small, rigid cylindrical elements such as large dumbbell-shaped molecules. The theory of micropolar fluids is generating a very much-increased interest \& many classical flows are being re-examined to determine the effects of the fluid microstructure [6].

Peddision \& McNitt (1970) applied the micropolar boundary layer theory to the problems of steady stagnation point flow, steady flow over a semi-infinite flat plate [10]. Gorla (1992) investigated the steady boundary layer flow of a micropolar fluid at a two dimensional stagnation point on a moving wall and claimed that the micropolar fluid model is capable of predicting results which exhibit turbulent flow characteristics, although it is difficult to see how a steady laminar boundary layer flow could 'appear' to be turbulent [7]. Takhar \& Soundalgekar(1985) have studied the effects of suction $\&$ injection on the flow of a micropolar fluid past a continuously moving semi-infinite porous plate [14]. Mohammadein \& Gorla (1996) analyzed the effects of magnetic field on the laminar boundary layer mixed convection flow of a micropolar fluid over a horizontal plate [9]. However the work by Rees \& Bassom (1996) on the Blassius boundary layer flow over a flat plate suggests that much more information about the solution of boundary layer flows of a micropolar fluid can be obtained [12]. Char and Chang (1995) studied the laminar free convection heat transfer of a micropolar fluid past an arbitrary curved surface 
using the cubic spline collocation numerical method. They showed that the flow field admits similarity solutions when the power functions for the stream-wise variations of the wall temperature and the body shape configurations are constants, and their sum of them is equal to unity [2]. El-hakien et al. have studied about Joule heating effects on magnetohydrodynamics (MHD) free convection flow of a micropolar fluid in 1999 [3]. EI-Amin (2001) has studied about MHD free convection and mass transfer flow in micropolar fluid with constant suction. He was to find out the problem of free convection with mass transfer flow for a micropolar fluid bounded by a vertical infinite surface under the action of a transverse magnetic field [4]. Rahman \& Sattar (2006) have studied about MHD convective flow of a micropolar fluid past a continuously moving vertical porous plate in the presence of heat generation/absorption [11]. In this work they have extended the work of El-Arabawy (2003) to a MHD flow taking into account the effect of free convection \& micro rotation inertia term which has been neglected by El- Arabawy [5]. In 2012 Micropolar fluid behavior on steady MHD free convection and mass transfer through a porous medium with constant heat and mass fluxes has been studied numerically by Haque et al. [8]. They have used Nachtsheim-Swigert iteration technique as the main tool for the numerical approach.

Therefore the aim of this research is to investigate the micropolar fluid behavior on MHD free convection and mass transfers flow with constant heat and mass fluxes. The problem is solved by finite difference method in case of twodimensional steady flow and obtained results are shown graphically as well as in tabular form.

\section{Mathematical Formulation}

The generalized Continuity equation, Momentum equation, Angular Momentum equation, Energy equation and Concentration equation in three dimensional are formed on the basis of Ohm's law and Maxwell's equation with study of Magneto Fluid Dynamics (MFD). The steady MHD free convection and mass transfer flow of an electrically conducting viscous incompressible micropolar fluid through an infinite vertical plate $y=0$ has been considered. The flow is assumed to be in the $x$-direction which is taken along the plate in the upward direction and $y$-axis is normal to it. The microrotation is $G=(0,0, \Gamma)$, where $(0,0, \Gamma)$ be the components of microrotation and the component $\Gamma$ is the angular velocity acting in $z$-direction (the rotation of $\Gamma$ is in the $x y$ plane). The temperature and the species concentration at the plate are constantly raised from $T_{w}$ and $C_{w}$ to $T_{\infty}$ and $C_{\infty}$ respectively, which are thereafter maintained constant, where $T_{\infty}$ and $C_{\infty}$ are the temperature and species concentration of the uniform flow respectively. A uniform magnetic field $\mathrm{B}$ is imposed to the plate $(y=0)$ to be acting along $y$ axis which is assumed to be electrically non-conducting. The level of concentration of foreign mass is assumed high so that the thermal diffusion and mass diffusion are considered. The magnetic field is of the form $\mathrm{B}=\left(0, B_{0}, 0\right)$ and the magnetic lines of force are fixed relative to the fluid. The equation of conservation of charge $\nabla . \mathrm{J}=0$ gives $J_{y}=$ constant, where $\mathrm{J}=\left(J_{x}, J_{y}, J_{z}\right)$ is the current density, the direction of propagation is considered only along $y$ axis and does not have any variation along the $y$-axis and the derivative of $J$ with respect to $y$ namely $\frac{\partial J_{y}}{\partial y}=0$. Since the plate is electrically non-conducting, this constant is zero and hence $J_{y}=0$ at the plate and hence zero everywhere.

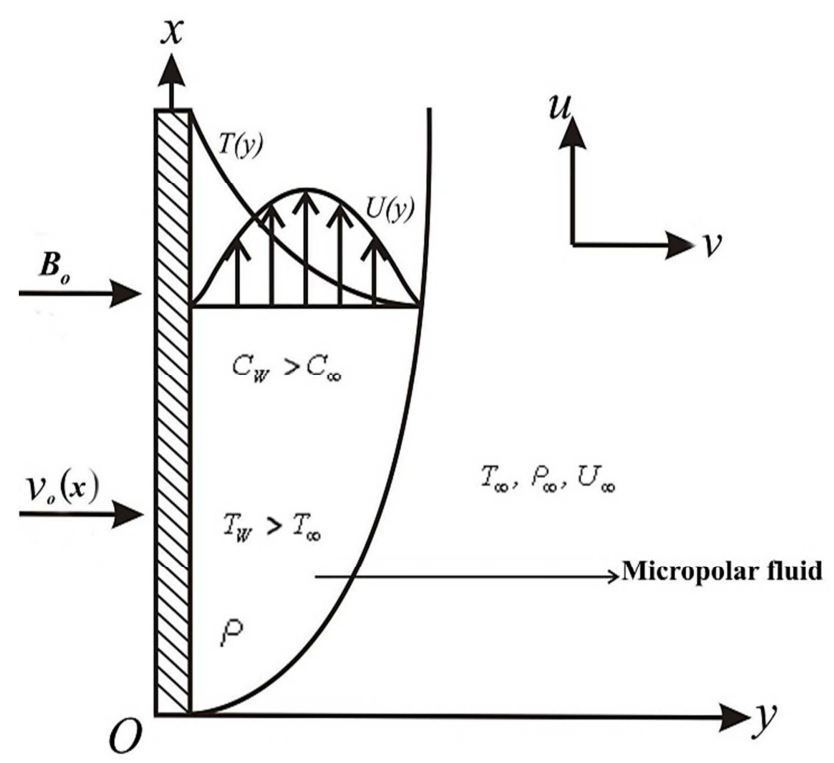

Fig. 1. Boundary layer development on a heated vertical plate.

With reference to the above assumptions, continuity equation, momentum equations, angular momentum equation, energy equation and concentration equation become

$$
\frac{\partial u}{\partial x}+\frac{\partial v}{\partial y}+\frac{\partial w}{\partial z}=0
$$

$$
\begin{gathered}
\frac{\partial u}{\partial t}+u \frac{\partial u}{\partial x}+v \frac{\partial u}{\partial y}+w \frac{\partial u}{\partial z}=F_{x}-\frac{1}{\rho} \frac{\partial P}{\partial x}+\left(v+\frac{\chi}{\rho}\right)\left(\frac{\partial^{2} u}{\partial x^{2}}+\frac{\partial^{2} u}{\partial y^{2}}+\frac{\partial^{2} u}{\partial z^{2}}\right)+\frac{\chi}{\rho} \frac{\partial \Gamma}{\partial y}-\frac{\sigma_{0}^{\prime} B_{0}^{2} u}{\rho} \\
\frac{\partial v}{\partial t}+u \frac{\partial v}{\partial x}+v \frac{\partial v}{\partial y}+w \frac{\partial v}{\partial z}=F_{y}-\frac{1}{\rho} \frac{\partial P}{\partial y}+\left(v+\frac{\chi}{\rho}\right)\left(\frac{\partial^{2} v}{\partial x^{2}}+\frac{\partial^{2} v}{\partial y^{2}}+\frac{\partial^{2} v}{\partial z^{2}}\right)+\frac{\chi}{\rho} \frac{\partial \Gamma}{\partial x}
\end{gathered}
$$




$$
\begin{gathered}
\frac{\partial w}{\partial t}+u \frac{\partial w}{\partial x}+v \frac{\partial w}{\partial y}+w \frac{\partial w}{\partial z}=F_{z}-\frac{1}{\rho} \frac{\partial P}{\partial z}+\left(v+\frac{\chi}{\rho}\right)\left(\frac{\partial^{2} w}{\partial x^{2}}+\frac{\partial^{2} w}{\partial y^{2}}+\frac{\partial^{2} w}{\partial z^{2}}\right) \\
\frac{\partial \Gamma}{\partial t}+u \frac{\partial \Gamma}{\partial x}+v \frac{\partial \Gamma}{\partial y}+w \frac{\partial \Gamma}{\partial z}=\frac{\gamma}{\rho j}\left(\frac{\partial^{2} \Gamma}{\partial x^{2}}+\frac{\partial^{2} \Gamma}{\partial y^{2}}+\frac{\partial^{2} \Gamma}{\partial z^{2}}\right)+\frac{\chi}{\rho j}\left(\frac{\partial v}{\partial x}-\frac{\partial u}{\partial y}\right)-2 \frac{\chi}{\rho j} \Gamma \\
\frac{\partial T}{\partial t}+u \frac{\partial T}{\partial x}+v \frac{\partial T}{\partial y}+w \frac{\partial T}{\partial z}=\frac{k}{\rho c_{p}}\left(\frac{\partial^{2} T}{\partial x^{2}}+\frac{\partial^{2} T}{\partial y^{2}}+\frac{\partial^{2} T}{\partial z^{2}}\right)+\frac{D_{m} k_{T}}{c_{s} c_{p}}\left(\frac{\partial^{2} C}{\partial x^{2}}+\frac{\partial^{2} C}{\partial y^{2}}+\frac{\partial^{2} C}{\partial z^{2}}\right) \\
\frac{\partial C}{\partial t}+u \frac{\partial C}{\partial x}+v \frac{\partial C}{\partial y}+w \frac{\partial C}{\partial z}=D_{m}\left(\frac{\partial^{2} C}{\partial x^{2}}+\frac{\partial^{2} C}{\partial y^{2}}+\frac{\partial^{2} C}{\partial z^{2}}\right)+\frac{D_{m} k_{T}}{c_{s} c_{p}}\left(\frac{\partial^{2} T}{\partial x^{2}}+\frac{\partial^{2} T}{\partial y^{2}}+\frac{\partial^{2} T}{\partial z^{2}}\right)
\end{gathered}
$$

Since the plate occupying the plane $y=0$ is of semiinfinite extent and the micropolar fluid motion is steady, all physical quantities will depend only upon $x$ and $y$. The boundary layer flow is driven by the buoyancy forces (Fig.1). The constant property conditions of the gravity force act in the negative $x$-direction. Also with one exception, assume the micropolar fluid to be incompressible. The exception involves accounting for the effect of variable density in the buoyancy force (so called Boussinesq's approximation), since it is the variation that induces micropolar fluid motion. The $x$-component momentum equation (2) reduces to the boundary layer equation if the only contribution to the body force is made by gravity, the body force per unit volume is $F_{x}=-\rho g$, where $\mathrm{g}$ is the local acceleration due to gravity. There is no body force in the $y$-direction $\left(\frac{\partial P}{\partial y}=0\right.$ i.e. $P=P(x)$ and $\left.F_{y}=0\right)$. Hence the $x$-pressure gradient at any point in the boundary layer must equal to the pressure gradient in the quiescent region outside the boundary layer. However, in this region $u=v=0$. Therefore the $x$ component of the momentum equation become $\frac{\partial P}{\partial x}=-\rho_{\infty} g$, where $\rho_{\infty}$ is the density of the surrounding fluid at temperature $T_{\infty}$. For small differences, the density difference term $\left(\rho-\rho_{\infty}\right)$ is related to the temperature and mass differences $\left(T-T_{\infty}\right)$ and $\left(C-C_{\infty}\right)$ respectively through the volume expansion coefficient $\beta$ and the volumetric coefficient $\beta^{*}$ by the relation

$$
\frac{\rho-\rho_{\infty}}{\rho}=-\beta\left(T-T_{\infty}\right)-\beta^{*}\left(C-C_{\infty}\right)
$$

Therefore

$$
F_{x}-\frac{1}{\rho} \frac{\partial P}{\partial x}=g \beta\left(T-T_{\infty}\right)+g \beta^{*}\left(C-C_{\infty}\right)
$$

Thus mathematically the problem reduces to a two dimensional problem. With the help of equation (8), the equations (1)-(7) become

$$
\begin{aligned}
& \frac{\partial u}{\partial x}+\frac{\partial v}{\partial y}=0 \\
& u \frac{\partial u}{\partial x}+v \frac{\partial u}{\partial y}=\left(v+\frac{\chi}{\rho}\right)\left(\frac{\partial^{2} u}{\partial x^{2}}+\frac{\partial^{2} u}{\partial y^{2}}\right)+g \beta\left(T-T_{\infty}\right) \\
& +g \beta^{*}\left(C-C_{\infty}\right)+\frac{\chi}{\rho} \frac{\partial \Gamma}{\partial y}-\frac{\sigma^{\prime} B_{0}{ }^{2} u}{\rho} \\
& u \frac{\partial v}{\partial x}+v \frac{\partial v}{\partial y}=\left(v+\frac{\chi}{\rho}\right)\left(\frac{\partial^{2} v}{\partial x^{2}}+\frac{\partial^{2} v}{\partial y^{2}}\right)-\frac{\chi}{\rho} \frac{\partial \Gamma}{\partial x} \\
& u \frac{\partial \Gamma}{\partial x}+v \frac{\partial \Gamma}{\partial y}=\frac{\gamma}{\rho j}\left(\frac{\partial^{2} \Gamma}{\partial x^{2}}+\frac{\partial^{2} \Gamma}{\partial y^{2}}\right)+\frac{\chi}{\rho j}\left(\frac{\partial v}{\partial x}-\frac{\partial u}{\partial y}\right)-2 \frac{\chi}{\rho j} \Gamma \\
& u \frac{\partial T}{\partial x}+v \frac{\partial T}{\partial y}=\frac{k}{\rho c_{p}}\left(\frac{\partial^{2} T}{\partial x^{2}}+\frac{\partial^{2} T}{\partial y^{2}}\right)+\frac{D_{m} k_{T}}{c_{s} c_{p}}\left(\frac{\partial^{2} C}{\partial x^{2}}+\frac{\partial^{2} C}{\partial y^{2}}\right) \\
& u \frac{\partial C}{\partial x}+v \frac{\partial C}{\partial y}=D_{m}\left(\frac{\partial^{2} C}{\partial x^{2}}+\frac{\partial^{2} C}{\partial y^{2}}\right)+\frac{D_{m} k_{T}}{c_{s} c_{p}}\left(\frac{\partial^{2} T}{\partial x^{2}}+\frac{\partial^{2} T}{\partial y^{2}}\right)
\end{aligned}
$$

The viscosity of the fluid and the small thickness of the boundary layer $\delta$ are considered to be small. Let $\varepsilon<<1$ be the order of magnitude of $\delta$, i.e. $O(\delta)=\varepsilon<<1$. Let the order of magnitude of $u, x$ and $\Gamma$ are one, i.e. $O(u)=1$, $O(x)=1, O(\Gamma)=1$. The order of magnitude of $v$ and $y$ are considered to be $\varepsilon$ i.e., $O(v)=\varepsilon, O(y)=\varepsilon$.

$$
\begin{aligned}
& \text { Hence } O\left(\frac{\partial u}{\partial x}\right)=1, O\left(\frac{\partial u}{\partial y}\right)=\frac{1}{\varepsilon}, O\left(\frac{\partial^{2} u}{\partial y^{2}}\right)=\frac{1}{\varepsilon^{2}} \text { and } \\
& O\left(\frac{\partial v}{\partial t}\right)=\varepsilon, O\left(\frac{\partial v}{\partial x}\right)=\varepsilon, O\left(\frac{\partial v}{\partial y}\right)=1, O\left(\frac{\partial^{2} v}{\partial y^{2}}\right)=\frac{1}{\varepsilon}, \\
& O\left(\frac{\partial \Gamma}{\partial x}\right)=1, O\left(\frac{\partial \Gamma}{\partial y}\right)=\frac{1}{\varepsilon}, O\left(\frac{\partial^{2} \Gamma}{\partial y^{2}}\right)=\frac{1}{\varepsilon^{2}} \text { within the }
\end{aligned}
$$

boundary layer. Since the boundary layer and the thermal boundary layer are same in this case, let $\delta$ be the thermal boundary layer thickness and $\varepsilon<<1$ be the order of magnitude of $\delta$, i.e., $O(\delta)=\varepsilon<<1$. Let the order of 
magnitude of $T$ and $C$ are one, i.e., $O(T)=1$.

Hence $O\left(\frac{\partial T}{\partial x}\right)=1 \quad, \quad O\left(\frac{\partial^{2} T}{\partial x^{2}}\right)=1 \quad, \quad O\left(\frac{\partial T}{\partial y}\right)=\frac{1}{\varepsilon}$, $O\left(\frac{\partial^{2} T}{\partial y^{2}}\right)=\frac{1}{\varepsilon^{2}}$ and $O\left(\frac{\partial C}{\partial x}\right)=1, O\left(\frac{\partial^{2} C}{\partial x^{2}}\right)=1, O\left(\frac{\partial C}{\partial y}\right)=\frac{1}{\varepsilon}$, $O\left(\frac{\partial^{2} C}{\partial y^{2}}\right)=\frac{1}{\varepsilon^{2}}$ within the boundary layer.

The steady two dimensional MHD free convection and mass transfer micropolar fluid flow, past a semi-infinite vertical porous plate is considered. The $x$-axis is taken along the heated plate in the upward direction and the $y$-axis normal to it. The plate is immersed in a micropolar fluid of temperature $T$. A magnetic field $\mathrm{B}$ of uniform strength is applied transversely to the direction of the flow. The magnetic Reynolds number of the flow is taken to be sufficiently small enough, so that the induced magnetic field can be neglected in comparison with applied magnetic field so that $\mathrm{B}=\left(0, B_{0}, 0\right)$, where $B_{0}$ is the uniform magnetic field acting normal to the plate. The flow configuration and the co-ordinate system are shown Fig.1. Within the frame work of the above noted assumptions, the flow of a steady viscous incompressible micropolar fluid flow subjected to the Boussinesq approximation can be written in the following form:

Continuity Equation

$$
\frac{\partial u}{\partial x}+\frac{\partial v}{\partial y}=0
$$

Momentum equation

$$
\begin{aligned}
u \frac{\partial u}{\partial x}+v \frac{\partial u}{\partial y} & =\left(v+\frac{\chi}{\rho}\right) \frac{\partial^{2} u}{\partial y^{2}}+\frac{\chi}{\rho} \frac{\partial \Gamma}{\partial y}+g \beta\left(T-T_{\infty}\right) \\
& +g \beta^{*}\left(C-C_{\infty}\right)-\frac{\sigma^{\prime} B_{0}^{2} u}{\rho}
\end{aligned}
$$

Angular momentum equation

$$
u \frac{\partial \Gamma}{\partial x}+v \frac{\partial \Gamma}{\partial y}=\frac{\gamma}{\rho j} \frac{\partial^{2} \Gamma}{\partial y^{2}}-\frac{\chi}{\rho j}\left(2 \Gamma+\frac{\partial u}{\partial y}\right)
$$

Energy equation

$$
u \frac{\partial T}{\partial x}+v \frac{\partial T}{\partial y}=\frac{\kappa}{\rho c_{p}} \frac{\partial^{2} T}{\partial y^{2}}+\frac{D_{m} \kappa_{T}}{c_{p} c_{s}} \frac{\partial^{2} C}{\partial y^{2}}
$$

Concentration equation

$$
u \frac{\partial C}{\partial x}+v \frac{\partial C}{\partial y}=D_{m} \frac{\partial^{2} C}{\partial y^{2}}+\frac{D_{m} \kappa_{T}}{c_{p} c_{s}} \frac{\partial^{2} T}{\partial y^{2}}
$$

The boundary conditions for the problem are:

at $t=0, u=0, v=0, \Gamma=0, T=0, C=0$ everywhere

$$
t>0 \begin{cases}u=0, v=0, \Gamma=0, T=0, C=0 & \text { at } \mathrm{x}=0 \\ u=0, v=0, \Gamma=0, T=1, C=1 & \text { at } \mathrm{y}=0 \\ u=0, v=0, \Gamma=0, T=0, C=0 & \text { at } \mathrm{y} \rightarrow \infty\end{cases}
$$

where $u, v$ are the velocity components in the $x, y$ direction respectively, $v$ is the kinematic viscosity, $g$ is the acceleration due to gravity, $\rho$ is the density, $\beta$ is the coefficient of volume expansion, $\beta^{*}$ is the volumetric coefficient of expansion with concentration. $T, T_{w}$ and $T_{\infty}$ are the temperature of the fluid inside the thermal boundary layer, the plate temperature and the fluid temperature in the free stream, respectively, while $\mathrm{C}, C_{w}, C_{\infty}$ are the corresponding concentrations. Also $\kappa$ is the thermal conductivity of the medium, $Q$ is the constant heat flux per unit area, $D_{m}$ is the coefficient of mass diffusivity, $c_{p}$ is the specific heat at constant pressure, $T_{m}$ is the mean fluid temperature, $c_{s}$ is the concentration susceptibility, $\sigma^{\prime}$ is the electrical conductivity, $\Gamma$ is the microrotation component, $\chi$ is the vortex viscosity, $\gamma$ is the spin gradient viscosity, $j$ is the microinertia per unit mass, $m$ is the coefficient of mass flux per unit area and $s$ is a constant (when $s=0$, we obtain $\Gamma=0$ which represents no-spin condition i.e., the microelements in a concentrated particle flow close to the wall are not able to rotate. The case $s=1 / 2$ represents vanishing of the anti-symmetric part of the stress tensor and represents weak concentration. In a fine particle suspension of the particle spin is equal to the fluid velocity at the wall. The case $s=1$ represents turbulent boundary layer flow) and other symbols have their usual meaning.

\section{Solution Technique}

Since the solutions of the governing equations (15)-(19) under the initial conditions (20) \& (21) will be based on the finite difference method it is required to make the said equations dimensionless. For this purpose we now introduce the following dimensionless quantities:

$$
\begin{aligned}
& X=\frac{x U_{o}}{v}, Y=\frac{y U_{o}}{v}, U=\frac{u}{U_{o}}, V=\frac{v}{U_{o}}, \tau=\frac{t U_{o}^{2}}{v}, \\
& \Gamma=\frac{\bar{\Gamma} U_{0}^{2}}{v}, \bar{T}=\frac{T-T_{\infty}}{T_{w}-T_{\infty}}, \bar{C}=\frac{C-C_{\infty}}{C_{w}-C_{\infty}}
\end{aligned}
$$

Using these relations we have the following dimensionless equations

$$
\begin{gathered}
\frac{\partial U}{\partial X}+\frac{\partial V}{\partial Y}=0 \\
\frac{\partial U}{\partial \tau}+U \frac{\partial U}{\partial X}+V \frac{\partial U}{\partial Y}=(1+\Delta) \frac{\partial^{2} U}{\partial Y^{2}}+\Delta \frac{\partial \bar{\Gamma}}{\partial Y}+G_{r} \bar{T}+G_{m} \bar{C}+M U
\end{gathered}
$$




$$
\begin{gathered}
\frac{\partial \bar{\Gamma}}{\partial \tau}+U \frac{\partial \bar{\Gamma}}{\partial X}+V \frac{\partial \bar{\Gamma}}{\partial Y}=\Lambda \frac{\partial^{2} \bar{\Gamma}}{\partial Y^{2}}-2 \lambda \bar{\Gamma}-\lambda \frac{\partial U}{\partial Y} \\
\frac{\partial \bar{T}}{\partial \tau}+U \frac{\partial \bar{T}}{\partial X}+V \frac{\partial \bar{T}}{\partial Y}=\frac{1}{P_{r}} \frac{\partial^{2} \bar{T}}{\partial Y^{2}}+E_{c}(1+\Delta)\left(\frac{\partial U}{\partial Y}\right)^{2}+D_{f} \frac{\partial^{2} \bar{C}}{\partial Y^{2}} \\
\frac{\partial \bar{C}}{\partial \tau}+U \frac{\partial \bar{C}}{\partial X}+V \frac{\partial \bar{C}}{\partial Y}=\frac{1}{S_{c}} \frac{\partial^{2} \bar{C}}{\partial Y^{2}}+S_{0} \frac{\partial^{2} \bar{T}}{\partial Y^{2}}
\end{gathered}
$$

With boundary conditions

$$
\begin{gathered}
\tau=0, U=0, V=0, \bar{\Gamma}=0, \bar{T}=0, \bar{C}=0 \text { everywhere } \\
\tau>0 \begin{cases}U=0, V=0, \bar{\Gamma}=0, \bar{T}=0, \bar{C}=0 \quad \text { at } \mathrm{X}=0 \\
U=0, V=0, \bar{\Gamma}=0, \bar{T}=1, \bar{C}=1 \quad \text { at } \mathrm{Y}=0 \\
U=0, V=0, \bar{\Gamma}=0, \bar{T}=0, \bar{C}=0 \quad \text { at } \mathrm{Y} \rightarrow \infty\end{cases}
\end{gathered}
$$

We attempt to solve the governing second order nonlinear coupled dimensionless partial differential equations with the associated initial and boundary conditions. For solving a transient free convection flow with mass transfer past a semiinfinite plate, Callahan and Marner(1976) used the explicit finite difference method which is conditionally stable [1]. On the contrary, the same problem was studied by Soundalgekar and Ganesan(1980) by an implicit finite difference method which is unconditionally stable [13]. The only difference between the two methods is that the implicit method being unconditionally stable and is less expansive from the point of view of computer time. However, these two methods produced the same results.

From the concept of the above discussion, the explicit finite difference method has been used to solve equations (22)-(26) subject to the conditions given by (27) and (28).

To obtain the difference equations the region of the flow is divided into a grid or mesh of lines parallel to $X$ and $Y$ axis is taken along the plate and $Y$-axis is normal to the plate. Here we consider that the plate of height $X_{\max }(=100)$ i.e. $X$ varies from 0 to 100 and regard $Y_{\max }(=25)$ as corresponding to $Y \rightarrow \infty$ i.e. $Y$ varies from 0 to 25 . There are $m=125$ and $n=$ 125 grid spacing in the $X$ and $Y$ directions respectively. It is assumed that $\Delta X, \Delta Y$ are constant mesh sizes along $\mathrm{X}$ and $\mathrm{Y}$ directions respectively and taken as $\Delta X=0.8(0 \leq x \leq 100)$; $\Delta Y=0.2(0 \leq y \leq 25)$, with the smaller time-step $\Delta \tau=0.005$.

Now $\bar{U}, \Gamma^{\prime}, T^{\prime}$ and $C^{\prime}$ denote the values of $U, \bar{\Gamma}, \bar{T}$ and $\bar{C}$ at the end of a time-step respectively. Using the explicit finite difference approximation we get the difference equations in the following forms:

Continuity equation

$$
\frac{U_{i, j}-U_{i-1, j}}{\Delta X}+\frac{V_{i, j}-V_{i, j-1}}{\Delta Y}=0
$$

Momentum equation

$$
\begin{aligned}
& \frac{\bar{U}_{i, j}-U_{i, j}}{\Delta \tau}+U_{i, j} \frac{U_{i, j}-U_{i-1, j}}{\Delta X}+V_{i, j} \frac{U_{i, j+1}-U_{i, j}}{\Delta Y} \\
& =(1+\Delta) \frac{U_{i, j+1}-2 U_{i, j}+U_{i, j-1}}{(\Delta Y)^{2}} \\
& +\Delta \frac{\bar{\Gamma}_{i, j+1}-\bar{\Gamma}_{i, j}}{\Delta Y}+G_{r} \bar{T}_{i, j}+G_{m} \bar{C}_{i, j}+M U_{i, j}
\end{aligned}
$$

Angular momentum equation

$$
\begin{aligned}
& \frac{\Gamma_{i, j}^{\prime}-\bar{\Gamma}_{i, j}}{\Delta \tau}+U_{i, j} \frac{\bar{\Gamma}_{i, j}-\bar{\Gamma}_{i-1, j}}{\Delta X}+V_{i, j} \frac{\bar{\Gamma}_{i, j+1}-\bar{\Gamma}_{i, j}}{\Delta Y} \\
& =\Lambda \frac{\bar{\Gamma}_{i, j+1}-2 \bar{\Gamma}_{i, j}+\bar{\Gamma}_{i, j-1}}{(\Delta Y)^{2}} \\
& -2 \lambda \bar{\Gamma}_{i, j}-\lambda \frac{U_{i, j+1}-U_{i, j}}{\Delta Y}
\end{aligned}
$$

Energy equation

$$
\begin{aligned}
& \frac{T_{i, j}^{\prime}-\bar{T}_{i, j}}{\Delta \tau}+U_{i, j} \frac{\bar{T}_{i, j}-\bar{T}_{i-1, j}}{\Delta X}+V_{i, j} \frac{\bar{T}_{i, j+1}-\bar{T}_{i, j}}{\Delta Y} \\
& =\frac{1}{P_{r}} \frac{\bar{T}_{i, j+1}-2 \bar{T}_{i, j}+\bar{T}_{i, j-1}}{(\Delta Y)^{2}} \\
& +E_{c}(1+\Delta)\left(\frac{U_{i, j+1}-U_{i, j}}{\Delta Y}\right)^{2}+D_{f} \frac{\bar{C}_{i, j+1}-2 \bar{C}_{i, j}+\bar{C}_{i, j-1}}{(\Delta Y)^{2}}
\end{aligned}
$$

Concentration equation

$$
\begin{aligned}
& \frac{C_{i, j}^{\prime}-\bar{C}_{i, j}}{\Delta \tau}+U_{i, j} \frac{\bar{C}_{i, j}-\bar{C}_{i-1, j}}{\Delta X}+V_{i, j} \frac{\bar{C}_{i, j+1}-\bar{C}_{i, j}}{\Delta Y} \\
& =\frac{1}{S_{c}} \frac{\bar{C}_{i, j+1}-2 \bar{C}_{i, j}+\bar{C}_{i, j-1}}{(\Delta Y)^{2}} \\
& +S_{0} \frac{\bar{T}_{i, j+1}-2 \bar{T}_{i, j}+\bar{T}_{i, j-1}}{(\Delta Y)^{2}}
\end{aligned}
$$

And the initial and boundary conditions with the finite difference scheme are

$$
\begin{aligned}
& \tau=0 \quad U_{i, j}^{0}=0, \quad V_{i, j}^{0}=0, \quad \bar{\Gamma}_{i, j}^{0}=0, \quad \bar{T}_{i, j}^{0}=0, \quad \bar{C}_{i, j}^{0}=0 \quad(34) \\
& U_{0, j}^{n}=0, \quad V_{0, j}^{n}=0, \quad \bar{\Gamma}_{0, j}^{n}=0, \quad \bar{T}_{0, j}^{n}=0, \quad \bar{C}_{0, j}^{n}=0 \\
& \tau>0 U_{i, 0}^{n}=0, \quad V_{i, 0}^{n}=0, \quad \bar{\Gamma}_{i, 0}^{n}=0, \quad \bar{T}_{i, 0}^{n}=1, \quad \bar{C}_{i, 0}^{n}=1 \\
& U_{i, L}^{n}=0, \quad V_{i, L}^{n}=0, \quad \bar{\Gamma}_{i, L}^{n}=0, \quad \bar{T}_{i, L}^{n}=0, \quad \bar{C}_{i, L}^{n}=0 \\
& \text { Where, } \quad L \rightarrow \infty
\end{aligned}
$$

Here the subscripts $i$ and $j$ designate the grid points with $x$ and $y$ coordinates respectively and the superscript $n$ represents a value of time, $\tau=n \Delta \tau$ where $n=0,1,2, \ldots .$. From the initial condition (34), the values of $U, \bar{\Gamma}, \bar{T}$ and $\bar{C}$ are known at $\tau=0$. During any one time-step, the 
coefficients $U_{i, j}$ and $V_{i, j}$ appearing in equations (29)-(33) are treated as constants. Then the end of anytime step $\Delta \tau$, the new velocity $\bar{U}$, the new temperature $T^{\prime}$, the new microrotation $\Gamma^{\prime}$ the new concentration $C^{\prime}$ at all interior nodal points be obtained by successive applications of equations (30), (31), (32) and (33) respectively. This process is repeated in time and provided the time-step is sufficiently small, $U, V, \bar{\Gamma}, \bar{T}$ and $\bar{C}$ should eventually converge to values which approximate the steady state solution of equations (22)-(26).

\section{Results and Discussions}

In this paper, the effects of Micropolar fluid behavior on MHD free convection and mass transfer flow with constant heat and mass fluxes have been investigated using the finite difference technique. To study the physical situation of this problem, we have computed the numerical values by finite different technique of velocity, micrirotation, temperature and concentration at the plate. It can be seen that the solutions are affected by the parameters namely, Microrotation parameter $(\Delta)$, Spin gradient viscosity parameter $(\Lambda)$ and the vortex viscosity parameter $(\lambda)$, Grashof Number $\left(G_{r}\right)$, Modified Grashof Number $\left(G_{m}\right)$, Prandtl number $\left(P_{r}\right)$, Magnetic parameter $(M)$, Eckert Number $\left(E_{c}\right)$, Dufour Number $\left(D_{f}\right)$, Schimidt Number $\left(S_{c}\right)$, and Soret Number $\left(S_{o}\right)$. The main goal of the computation is to obtain the steady state solutions for the non-dimensional velocity $U$, temperature $T^{\prime}$, concentration $C^{\prime}$ and Microrotation $\Gamma^{\prime}$ for different values of Microrotation parameter $(\Delta)$, Spin gradient viscosity parameter $(\Lambda)$ and the vortex viscosity parameter $(\lambda)$, Grashof Number $\left(G_{r}\right)$, Modified Grashof Number $\left(G_{m}\right)$, Prandtl Number $\left(P_{r}\right)$,

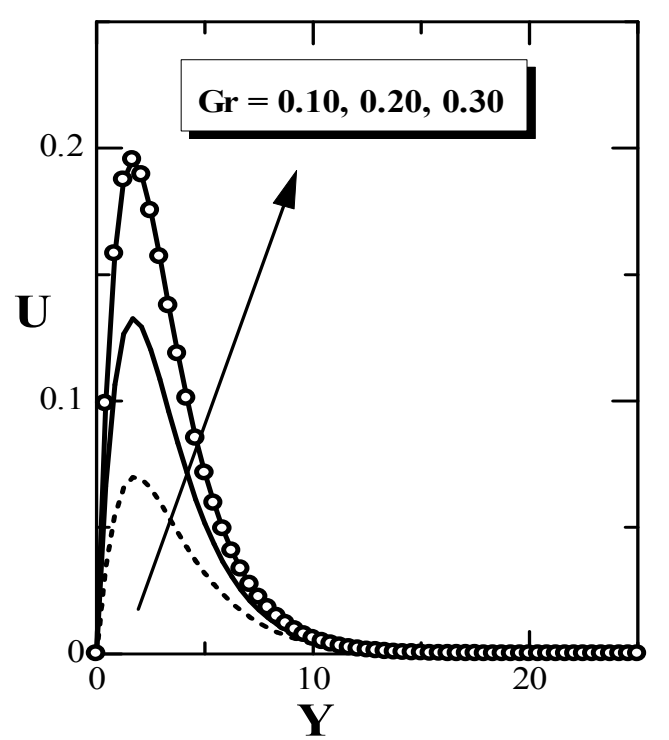

Fig. 2. Velocity profile for different values of Grashoff number $\left(G_{r}\right)$ at time $\tau=10$.
Magnetic parameter $(M)$, Eckert Number $\left(E_{c}\right)$, Dufour Number $\left(D_{f}\right)$, Schimidt Number $\left(S_{c}\right)$, and Soret Number $\left(S_{o}\right)$. For this computations the results have been calculated and presented graphically by dimensionless time $\tau=10$ up to $\tau=80$. The results of the computations show little changes for $\tau=10$ to $\tau=60$. But while arising at $\tau=70$ and 80 the results remain approximately same. Thus the solution for $\tau=80$ are become steady-state. Moreover, the steady state solutions for transient values of $U, T^{\prime}, C^{\prime}$ and $\Gamma^{\prime}$ are shown in figures (2-33), for time $\tau=10,20,30,40,50,60,70,80$ respectively. Figures (2-9) show the velocity profile for different values of Grashof Number $\left(G_{r}=0.10,0.20,0.30\right)$ at time $\tau=10,20,30,40,50,60,70,80$ respectively. From these figures are observed that the velocity increases with the increase of Grashof Number $\left(G_{r}\right)$. When it arising at $\tau=$ 70 and 80, the solutions become steady-state. The effects of temperature are shown in figures (10-17) for different values of Prandtl Number $\left(P_{r}\right)$, at time $\tau=10,20,30,40,50,60,70,80$ respectively. It is seen from these figures that the temperature decreases with the increase of Prandtl Number $\left(P_{r}\right)$. The concentration effects are shown in figures (18-25) for different values of Schmidt Number $\left(\mathrm{S}_{\mathrm{c}}\right)$, at time $\tau=10,20,30,40,50,60,70,80$ respectively and is clear from these figures that the concentration decreases with the increase of Schmidt Number $\left(\mathrm{S}_{\mathrm{c}}\right)$. The results remain approximately same while arising at time $\tau=70$ and 80 . Effects of microrotation are shown in Figures (26-33) for different values of Spin gradient viscosity parameter $(\Lambda)$ at time $\tau=10,20,30,40,50,60,70,80$ respectively. It is seen from these figures that the microrotation decreases with the increase of Spin gradient viscosity parameter $(\Lambda)$.

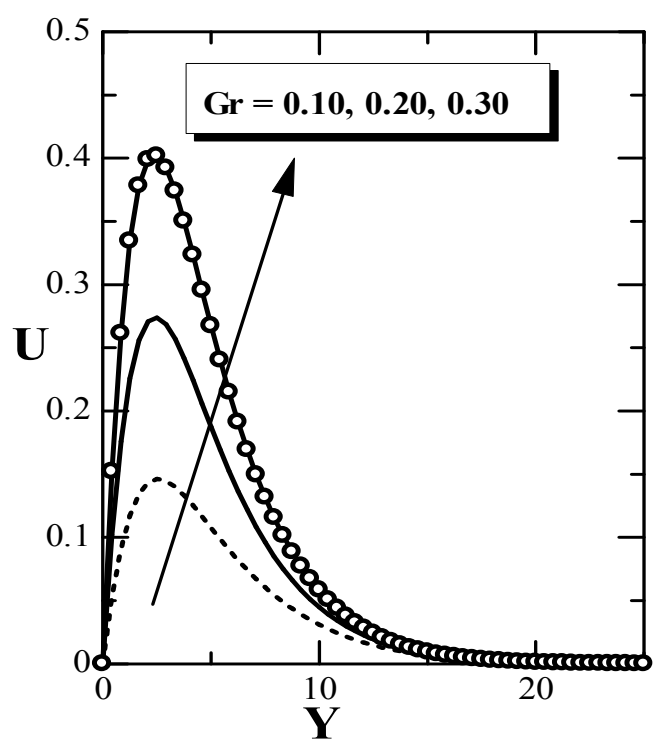

Fig. 3. Velocity profile for different values of Grashoff number $\left(G_{r}\right)$ at time $\tau=20$. 


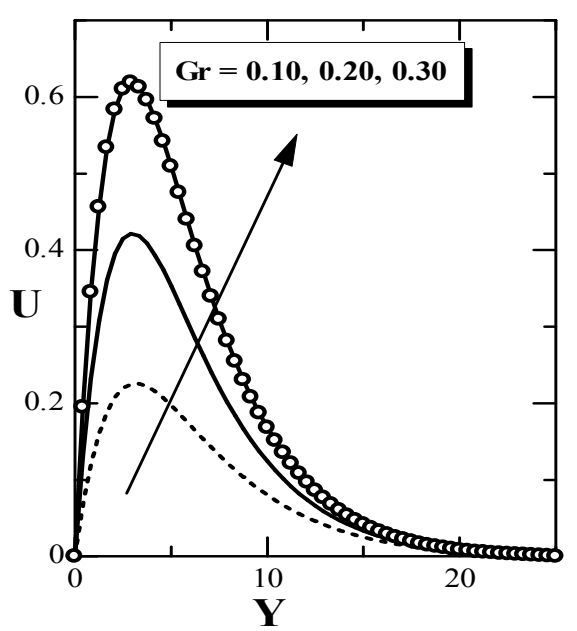

Fig. 4. Velocity profile for different values of Grashoff number $\left(G_{r}\right)$ at time $\tau=30$.

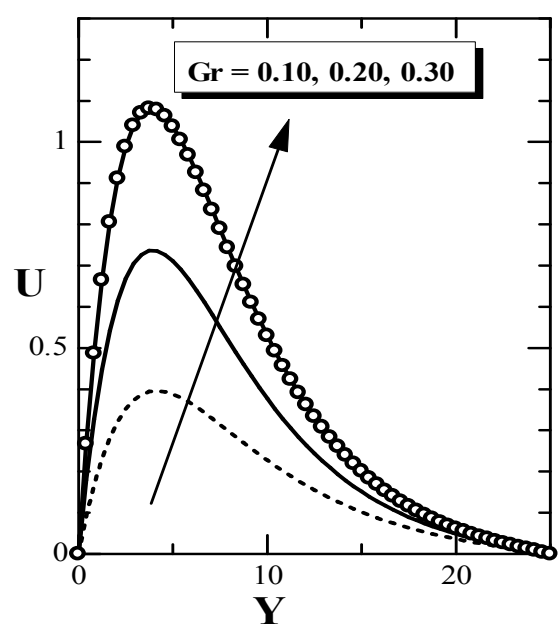

Fig. 6. Velocity profile for different values of Grashoff number $\left(G_{r}\right)$ at time $\tau=50$.

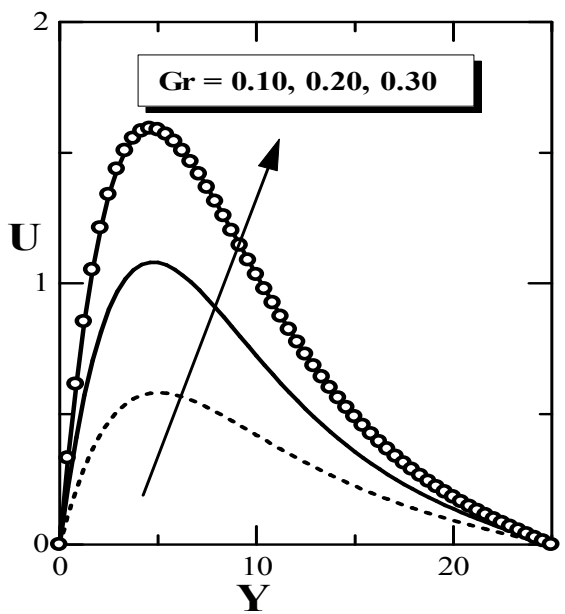

Fig. 8. Velocity profile for different values of Grashoff number $\left(G_{r}\right)$ at time $\tau=70$.

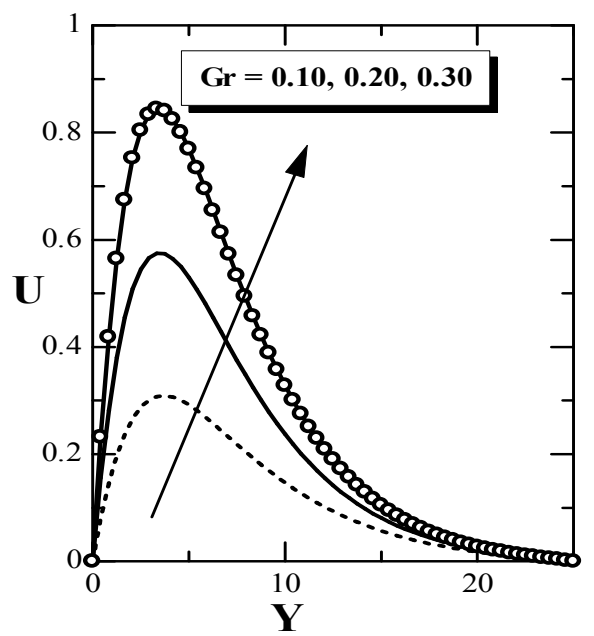

Fig. 5. Velocity profile for different values of Grashoff number $\left(G_{r}\right)$ at time $\tau=40$.

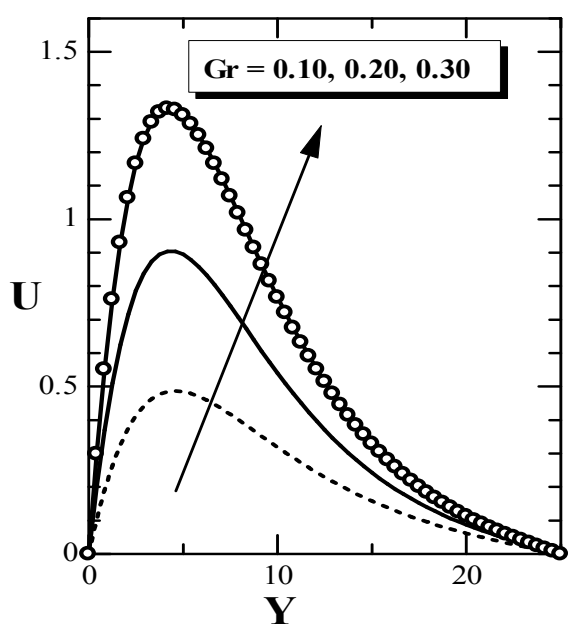

Fig. 7. Velocity profile for different values of Grashoff number $\left(G_{r}\right)$ at time $\tau=60$.

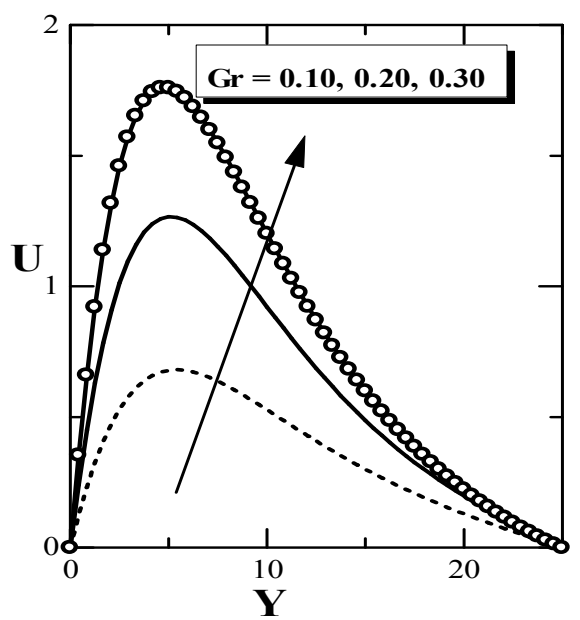

Fig. 9. Velocity profile for different values of Grashoff number $\left(G_{r}\right)$ at time $\tau=80$. 


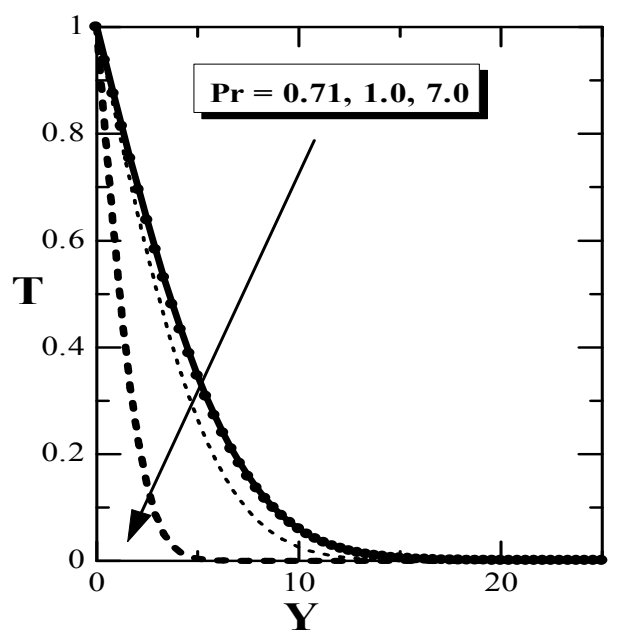

Fig. 10. Temperature profile for different values of Prandtle number $\left(P_{r}\right)$ at time $\tau=10$.

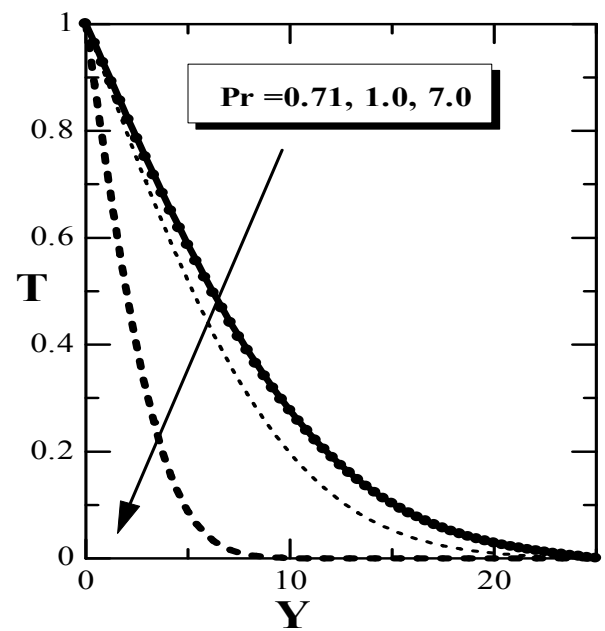

Fig. 12. Temperature profile for different values of Prandtle number $\left(P_{r}\right)$ at time $\tau=30$.

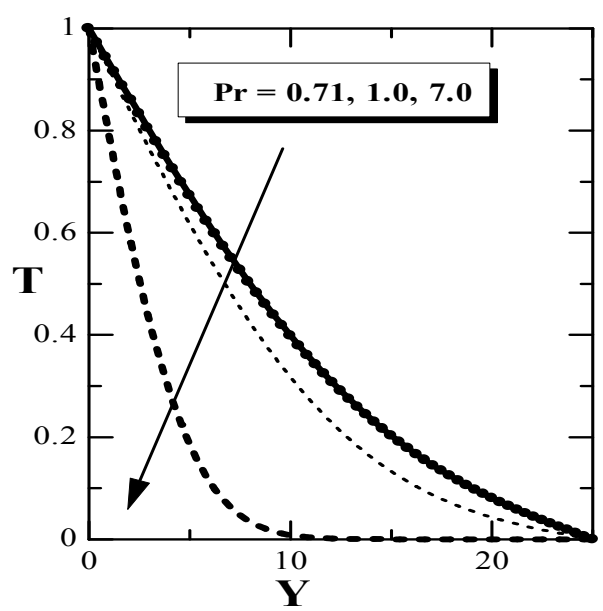

Fig. 14. Temperature profile for different values of Prandtle number $\left(P_{r}\right)$ at time $\tau=50$.

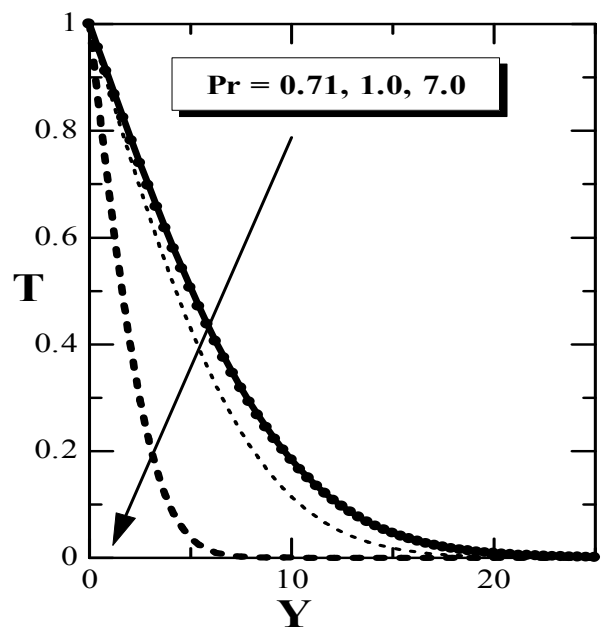

Fig. 11. Temperature profile for different values of Prandtle number $\left(P_{r}\right)$ at time $\tau=20$.

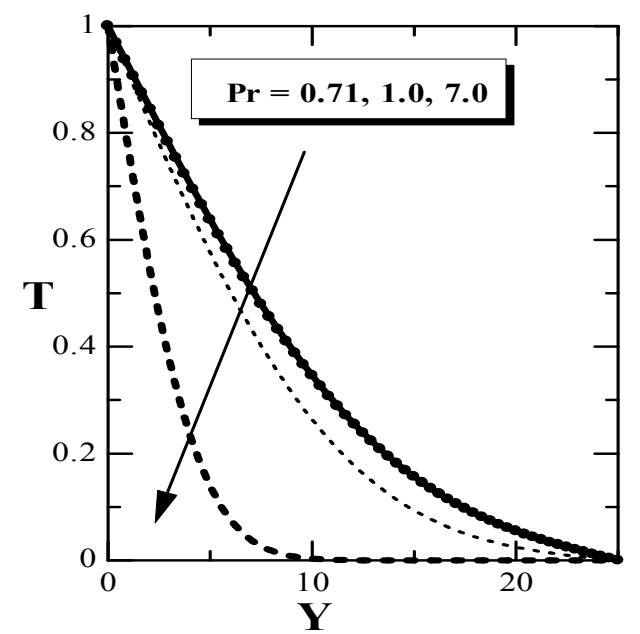

Fig. 13. Temperature profile for different values of Prandtle number $\left(P_{r}\right)$ at time $\tau=40$.

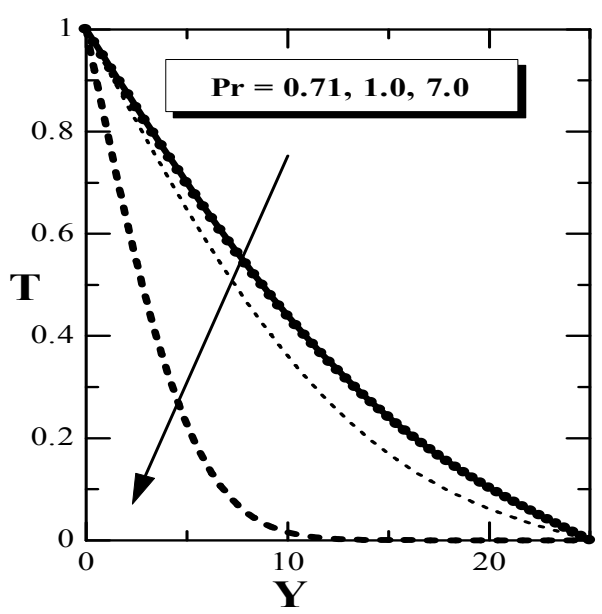

Fig. 15. Temperature profile for different values of Prandtle number $\left(P_{r}\right)$ at time $\tau=60$ 


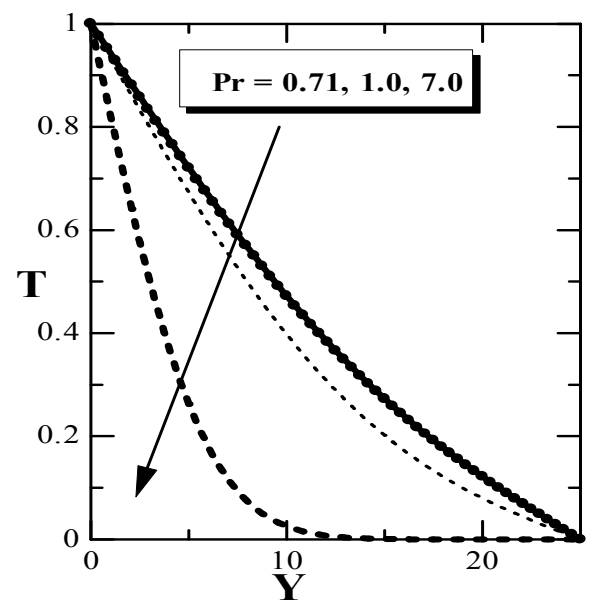

Fig. 16. Temperature profile for different values of Prandtle number $\left(P_{r}\right)$ at time $\tau=70$.

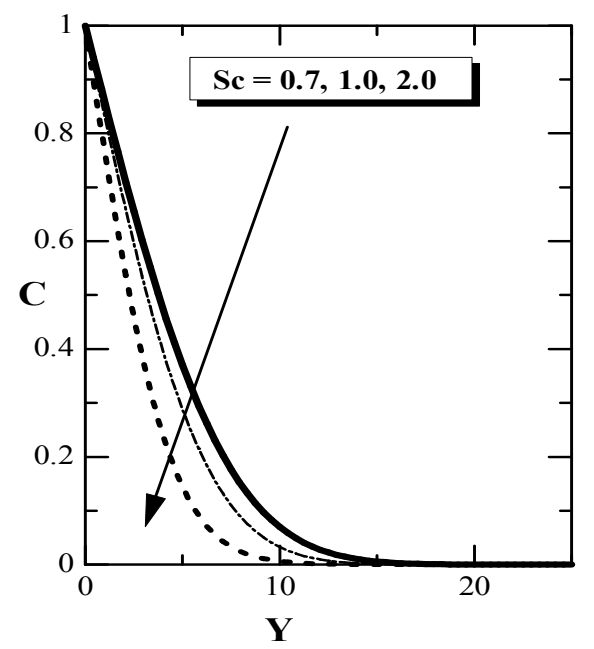

Fig. 18. Concentration profile for different values of Schmidt number $\left(S_{c}\right)$ at time $\tau$ $=10$.

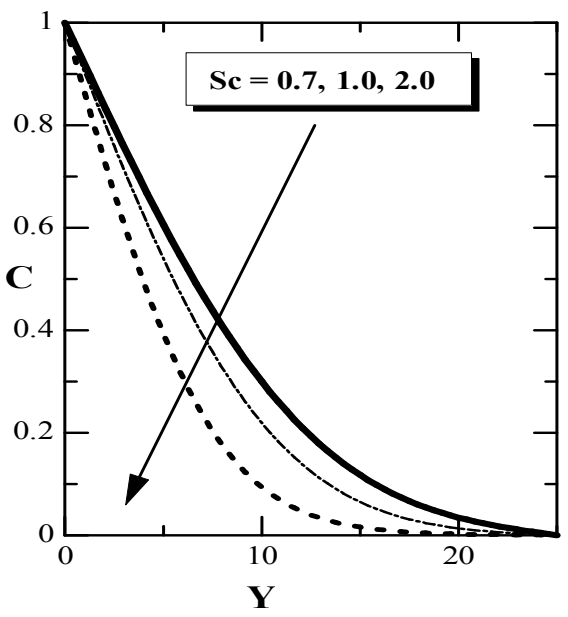

Fig. 20. Concentration profile for different values of Schmidt number $\left(S_{c}\right)$ at time $\tau$ $=30$.

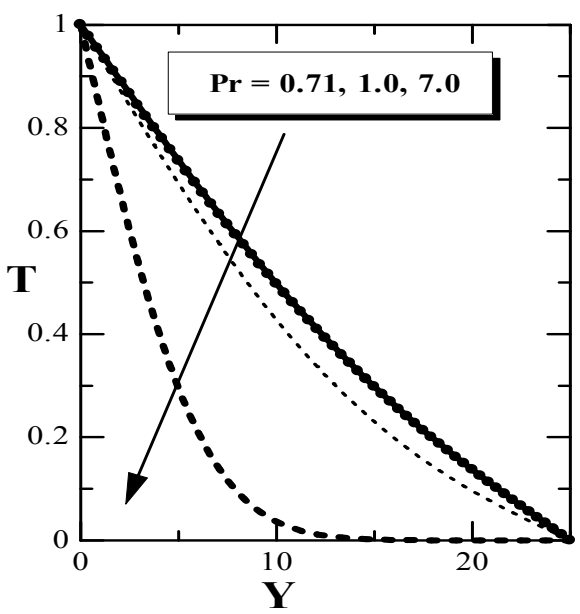

Fig. 17. Temperature profile for different values of Prandtle number $\left(P_{r}\right)$ at time $\tau=80$.

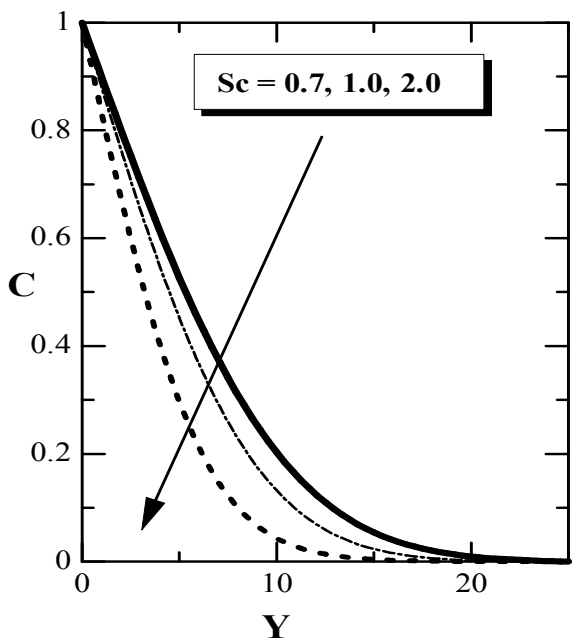

Fig. 19. Concentration profile for different values of Schmidt number $\left(S_{c}\right)$ at time $\tau=20$.

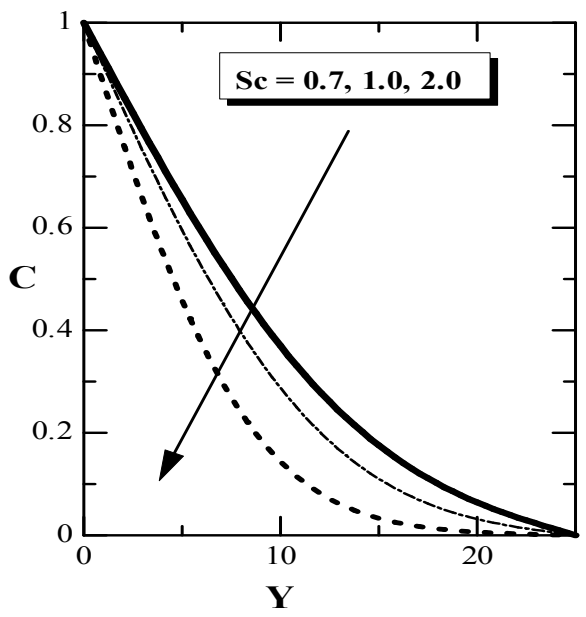

Fig. 21. Concentration profile for different values of Schmidt number $\left(S_{c}\right)$ at time $\tau=40$. 


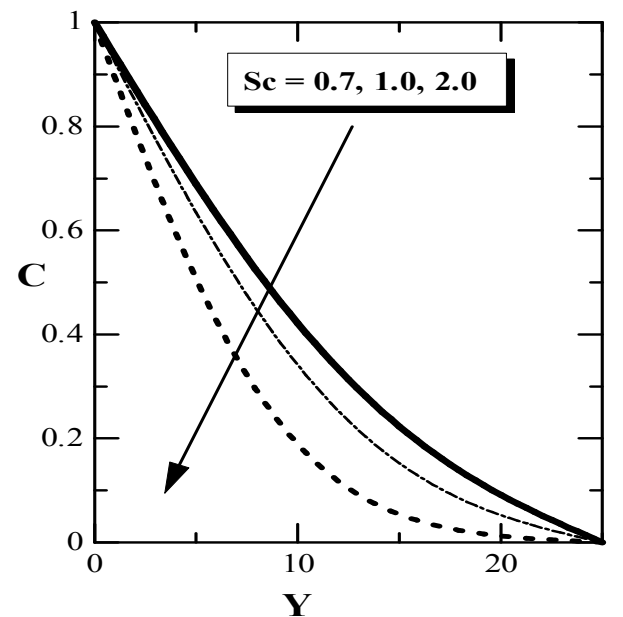

Fig. 22. Concentration profile for different values of Schmidt number $\left(S_{c}\right)$ at time $\tau$ $=50$.

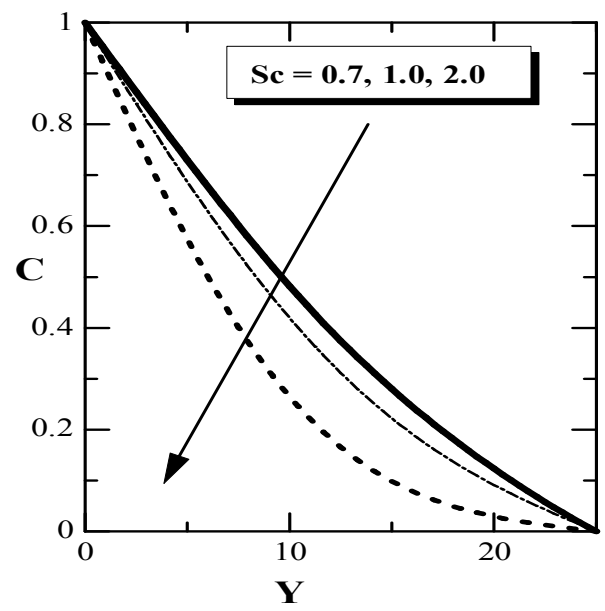

Fig. 24. Concentration profile for different values of Schmidt number $\left(S_{c}\right)$ at time $\tau$ $=70$.

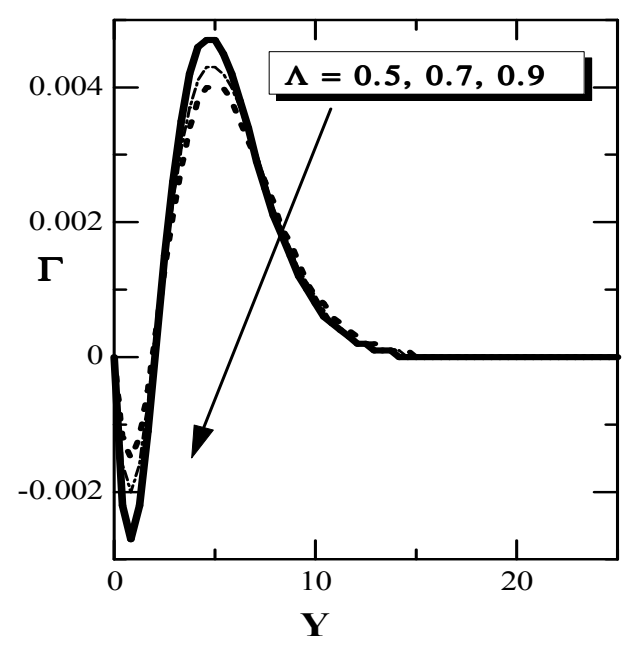

Fig. 26. Microrotation profile for different values of Spin gradient viscosity parameter $(\Lambda)$ at time $\tau=10$.

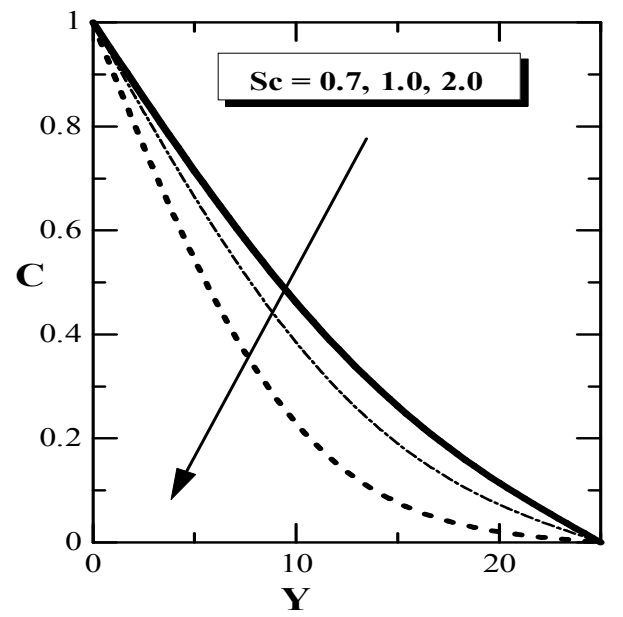

Fig. 23. Concentration profile for different values of Schmidt number $\left(S_{c}\right)$ at time $\tau=60$.

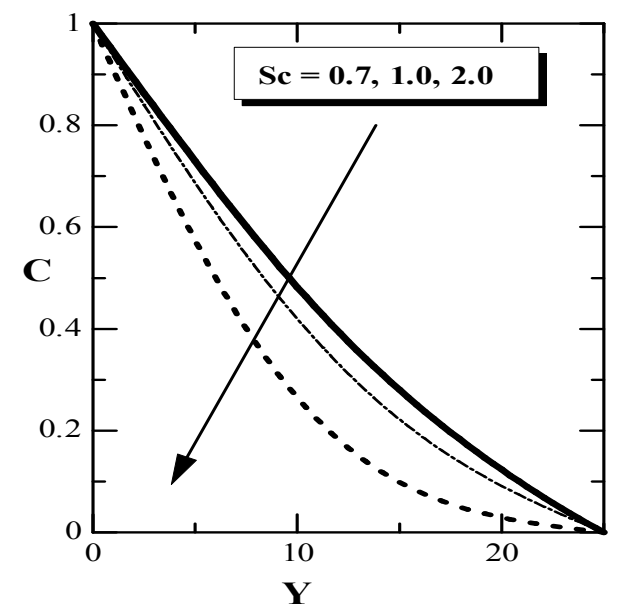

Fig. 25. Concentration profile for different values of Schmidt number $\left(S_{c}\right)$ at time $\tau=80$.

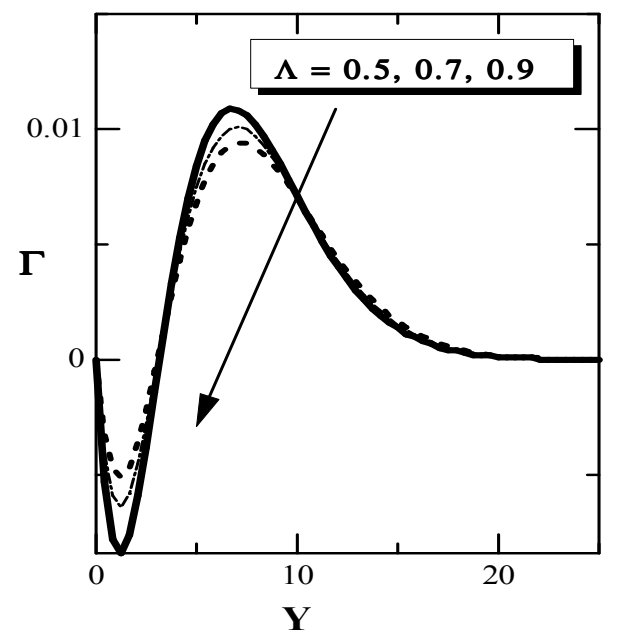

Fig. 27. Microrotation profile for different values of Spin gradient viscosity parameter $(\Lambda)$ at time $\tau=20$. 


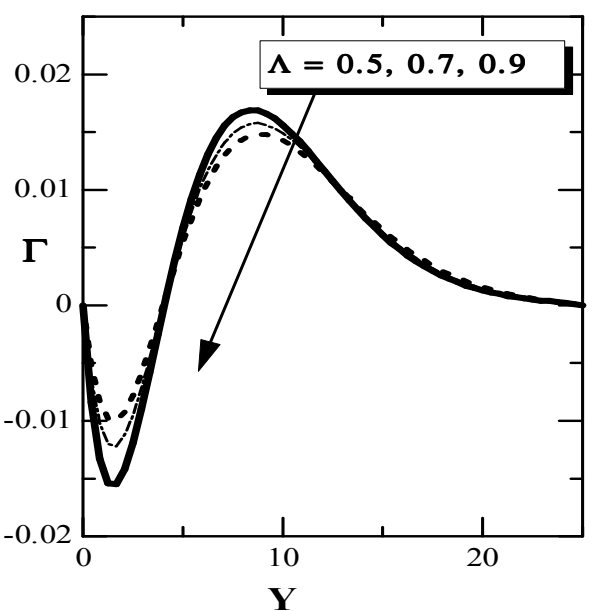

Fig. 28. Microrotation profile for different values of Spin gradient viscosity parameter $(\Lambda)$ at time $\tau=30$.

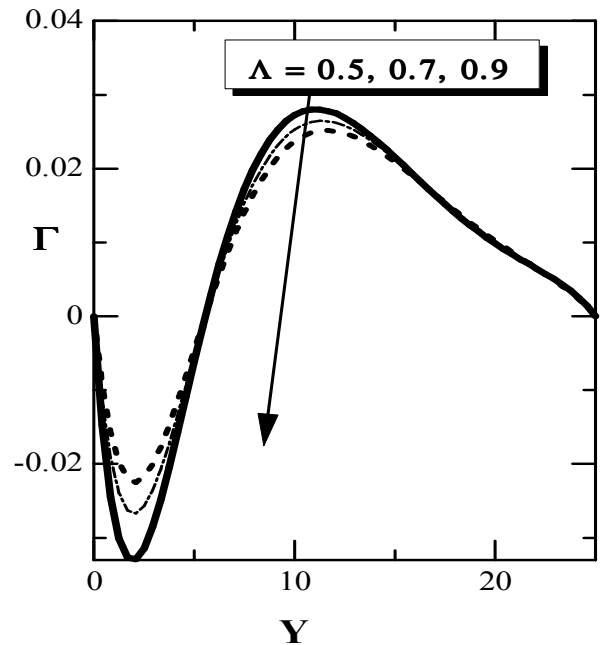

Fig. 30. Microrotation profile for different values of Spin gradient viscosity parameter $(\Lambda)$ at time $\tau=50$.

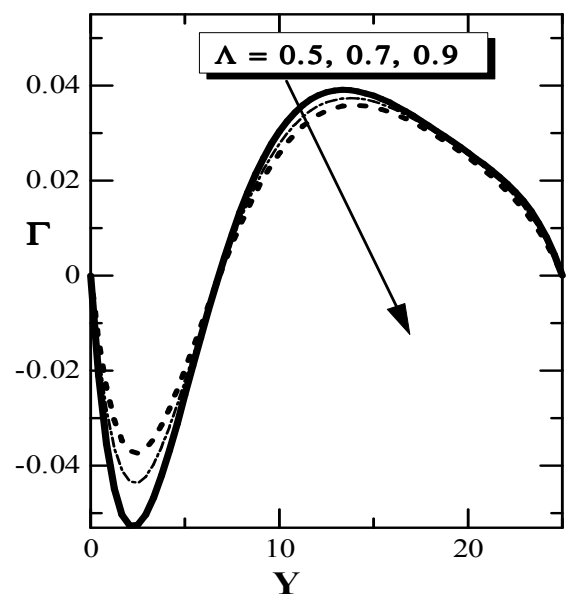

Fig. 32. Microrotation profile for different values of Spin gradient viscosity parameter $(\Lambda)$ at time $\tau=70$.

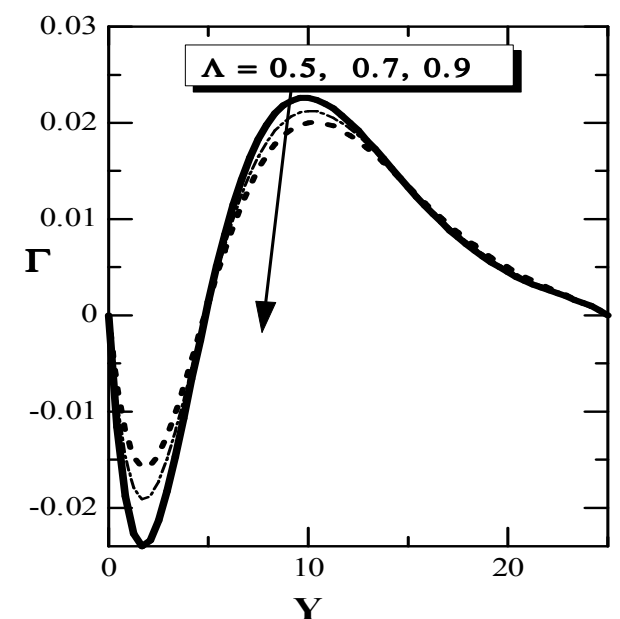

Fig. 29. Microrotation profile for different values of Spin gradient viscosity parameter $(\Lambda)$ at time $\tau=40$.

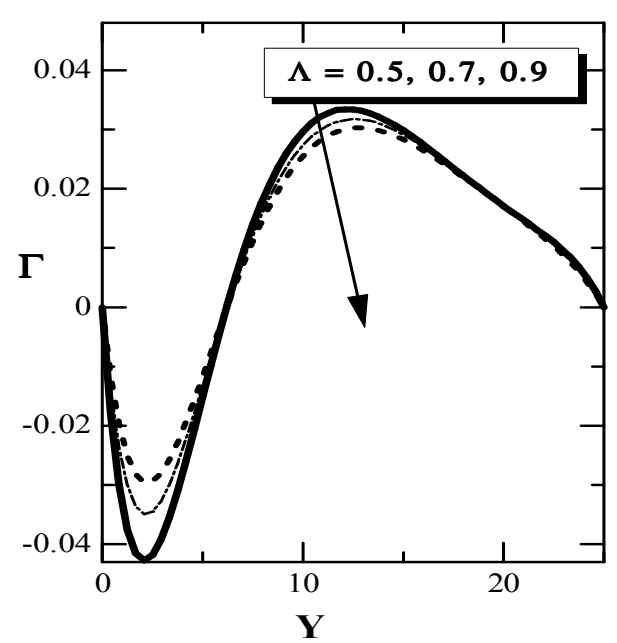

Fig. 31. Microrotation profile for different values of Spin gradient viscosity parameter $(\Lambda)$ at time $\tau=60$.

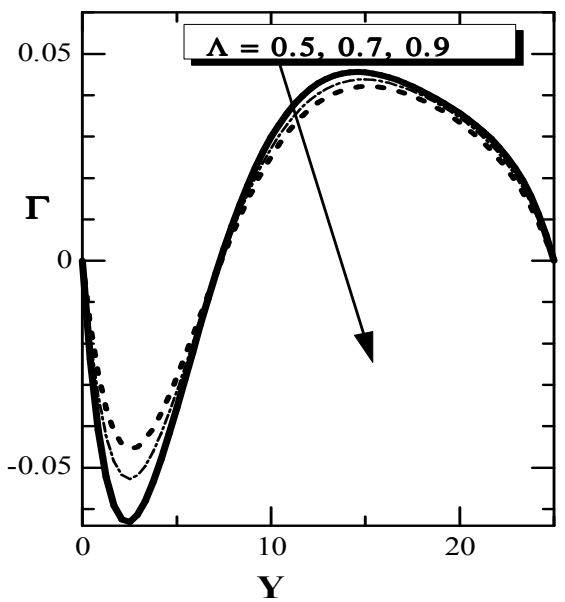

Fig. 33. Microrotation profile for different values of Spin gradient viscosity parameter $(\Lambda)$ at time $\tau=80$. 


\section{Conclusion}

Micropolar fluid behavior on MHD free convection and mass transfer flow with constant heat and mass fluxes have been investigated in this work. The resulting governing dimensionless coupled non-linear partial differential equations are numerically solved by an explicit finite difference method. The results are presented for different values of important parameters as the Grashoff Number, Prandtle numbe, Schmidt number and Spin gradient viscosity parameter with the fixed value of Modified Grashoff Number. The obtained important findings are listed below:

i The velocity profiles increase with the increase of Grashoff Number $\left(G_{r}\right)$. Particularly, the fluid velocity gradually increases with the increase of time.

ii The temperature profiles decrease with the increase of Prandtle number $\left(P_{r}\right)$. Particularly, due to increase of time the fluid temperature is shifted to upward direction.

iii The concentration profiles are also decrease with the increase of Schmidt number $\left(S_{c}\right)$. Particularly, due to increase of time the concentration is shifted to upward direction too.

iv Finally the Microrotation profiles decrease with the increase of Spin gradient viscosity parameter $(\Lambda)$.

\section{Acknowledgement}

This research project has supported by University Grant Commission (UGC), Bangladesh through Khulna University Research Cell. Therefore we have expressed our gratefulness to UGC and Khulna University Research Cell for the financial support and to Mathematics Discipline, Khulna University for providing Lab facilities.

\section{References}

[1] Callahan, G. D., and Marner, W. J., (1976), Transient free convection with mass transfer on an isothermal vertical flat plate. International Journal of Heat and Mass Transfer, 19:165-174.

[2] Char, M. I., and Chang, C. L., (1995), Laminar free convection flow of micropolar fluids from a curved surface. Journal of Physics D: Applied Physics, 28:1324-1331.
[3] El-Haikem, M. A., Mohammadein, A. A. and El-Kabeir, S. M. M., (1999), Joule heating effects on magneto hydrodynamic free convection flow of a micropolar fluid. International Journal of Communication Heat and Mass Transfer, 2:219227.

[4] El-Amin, M. F., (2001), Magneto hydrodynamic free convection and mass transfer flow in micropolar fluid with constant suction. Journal of Magnetism and Magnetic Materials, 234(3):567-574.

[5] El-Arabawy, H. A. M., (2003), Effect of suction/injection on the flow of a micropolar fluid past a continuously moving plate in the presence of radiation. International Journal of Heat and Mass Transfer, 46:1471-1477.

[6] Eringen, A. C., (1966), Theory of micropolar fluids. Journal of Mathematical Mechanics, 16:1-18.

[7] Gorla, R. S. R., (1992), Mixed convection in a micropolar fluid from a vertical surface with uniform heat flux. International Journal of Engineering and Science, 30:349358.

[8] Haque, M. Z., Alam, M. M., Ferdows, M., and Postelnicu, A., (2012), Micropolar fluid behaviors on steady MHD free convection and mass transfer flow with constant heat and mass fluxes, joule heating and viscous dissipation. Journal of King Saud University-Engineering Sciences, 24:71-84.

[9] Mohammadein, A. A. and Gorla R. S. R., (1996), Effects of transverse magnetic-field on mixed convection in a micropolar fluid on a horizontal plate with vectored mass-transfer. Acta Mechanica, 118:1-12.

[10] Peddiesen, J., and McNitt, R. P., (1970), Boundary layer theory for micropolar fluid. Recent Advanced Engineering Science, 5:405-426.

[11] Rahman, M. M., and Sattar, M. A., (2006), Magneto hydrodynamic convective flow of a micropolar fluid past a continuously moving porous plate in the presence of heat generation/absorption. ASME, Journal of Heat Mass Transfer, 128:142-152.

[12] Rees, D. A. S., and Bassom, A. P., (1996), The Blasius boundary layer flow of a micropolar fluid. International Journal of Engineering and Science, 34:113-124.

[13] Soundalgekar, V.M., and Ganesan, P., (1981), Finite difference analysis of transient free convection with mass transfer on an isothermal vertical flat plate. International Journal of Engineering and Science, 19:757-770.

[14] Takhar, H. S., and Soundalgekar, V. M., (1985), Flow and heat transfer of micropolar fluid past a porous plate. International Journal of Engineering and Science, 23:201-205. 